\title{
Effects of the Environment on Infectious Diseases
}

\author{
Dr. Noelle Samia \\ Northwestern University, \\ USA
}

In the keynote lecture, we address the effects of climate fluctuations on two areas of infectious diseases: bubonic plague and malaria. Plague (caused by the bacterium Yersinia pestis) is a zoonotic emerging infectious disease, with reservoirs in rodent populations worldwide. Using unique data on the main components of the host-vector-pathogen system as well as on its human victims, we discuss the multiple effects of climate forcing and climate change on the integral parts of the full eco-epidemiological plague system. In the case of malaria, we examine the effects of seasonality and environment on malaria incidence and we work towards accurately predicting the yearly peaks in malaria incidence as this is key for health systems to correctly anticipate an influx in malaria cases during certain months and most importantly, implement early prevention measures. 